\title{
Design of the vehicle driver fatigue monitor system based on ARM
}

\author{
Haichuan Han $^{1, a}$, Sanpeng Deng ${ }^{1, b}$ \\ ${ }^{1}$ Institute of Mechatronics Engineering, Tianjin University of Technology and Education, Tianjin, \\ 300222, China \\ ahaichuan_catarc@163.com, bsanpeng@yeah.net
}

Keywords: Fatigue driving, early warning system, multi-source information, embedded

\begin{abstract}
Fatigue driving is refers to the driver in a long time continuous driving or physical fatigue condition, and then come into being physiological and psychological function disorder, led to a decline in driving ability. Aimed at the requirements of monitoring on the fatigue driving, this article designed an driver fatigue monitor system based STM32F407 of ARM as a controller, it used to determine the driver's fatigue and reduce the traffic accident. The system adopts the method of multi-source information fusion, by monitoring the pulse, heart rate, temperature of the human body, steering wheel grip strength to realized the fatigue level. The system of graphical interface adopts UCGUI. Finally, testing the main function modules of early warning system, verify the feasibility of the proposed early warning system.
\end{abstract}

\section{Introduction}

With the rapid increase of motor vehicle quantities and the road mileage increased year by year, a large number of traffic accidents caused the world huge property losses and casualties. Each year 600000 deaths and economic losses of about $\$ 12.5$ billion caused by traffic accidents in the global, $57 \%$ of these accidents related to fatigue driving, Fatigue driving is the reaction levels drop after a period of time, and lead to can't normal driving, when driver is fatigue, its psychological state will also has a variety of changes, such as vision loss and causing distraction, slow in reacting, stiff action and arrhythmia, Therefore, the accurate monitoring of driver's fatigue state and implement effective early warning is of great significance to avoid fatigue driving effectively.

\section{Hardware design}

At present there are many research about the early-warning system of fatigue driving, But there's no a method was accepted by everyone. The main reason that although each method in one area is persuasive and operability, But it haven't reached the requirements of vehicle, real-time, objective, System with DSP kernel, the information data has high processing speed and good control performance of the 32-bit ARM processor STM32F407 as a controller, The 3.2 inch LCD screen with the small size, low power consumption as the human-computer interaction interface, figure 1 is system hardware structure diagram.

Car driver fatigue early warning system for embedded hardware mainly includes STM32F407 core processor, a 3.2 -inch LCD touch screen, data storage module, data communication interface module, information collection module, sensor module and alarm device. LCD touch screen based on the system of the monitoring results show that the current driver in a sober, mild or severe fatigue, fatigue and LCD touch screen is the driver and early warning system of human-computer interaction interface, The driver turned on or off using LCD touch screen early warning system for fatigue monitoring function, it can also view the current own pulse, heart rate, body temperature and other physical information.

The main functions of the system: through micro pulse sensor acquisition driver pulse signal; through a serial port communicate with tiny heart rate sensor acquisition drivers' heart rate signal; Through the infrared temperature sensor acquisition driver temperature signal; Through the thin film pressure sensor signal acquisition driver steering wheel grip; System collected the human body 
physiological signal data and preprocessed, then it displayed on the LCD panel, at the same time ti take the data stored in the storage module of the system.

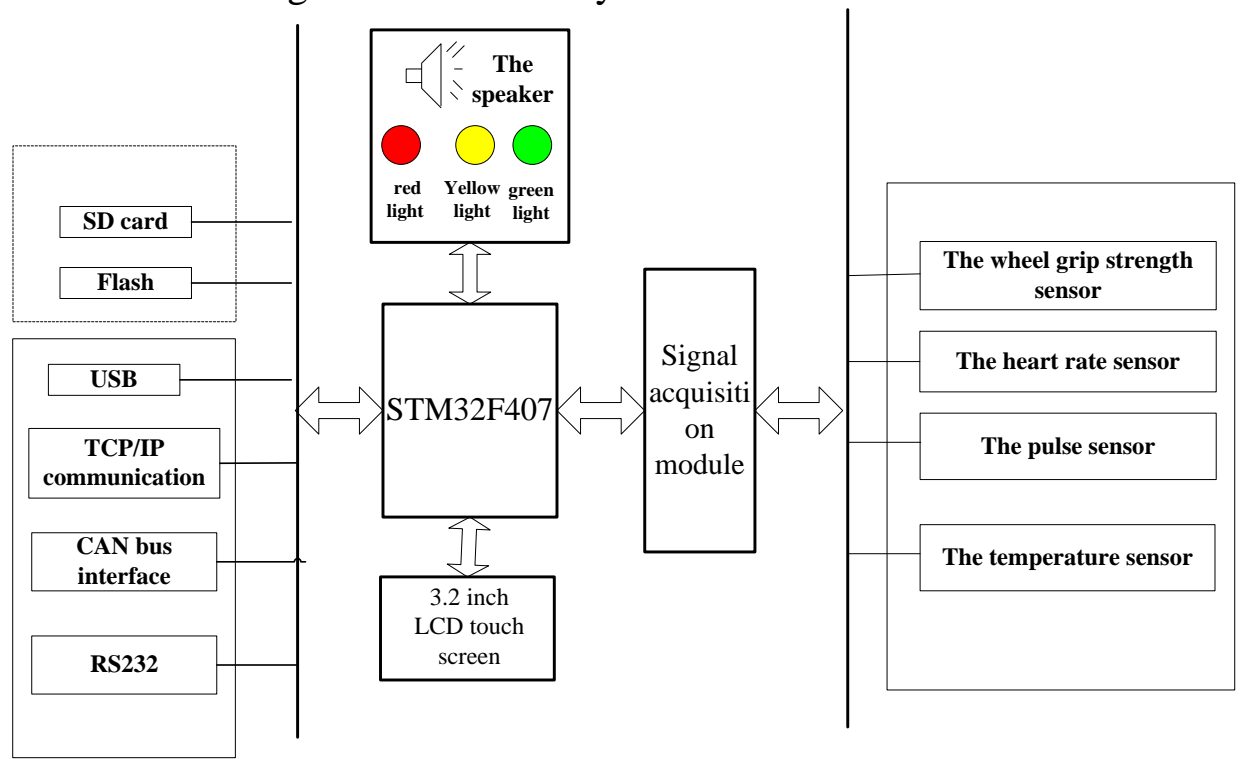

Fig. 1 System hardware structure diagram

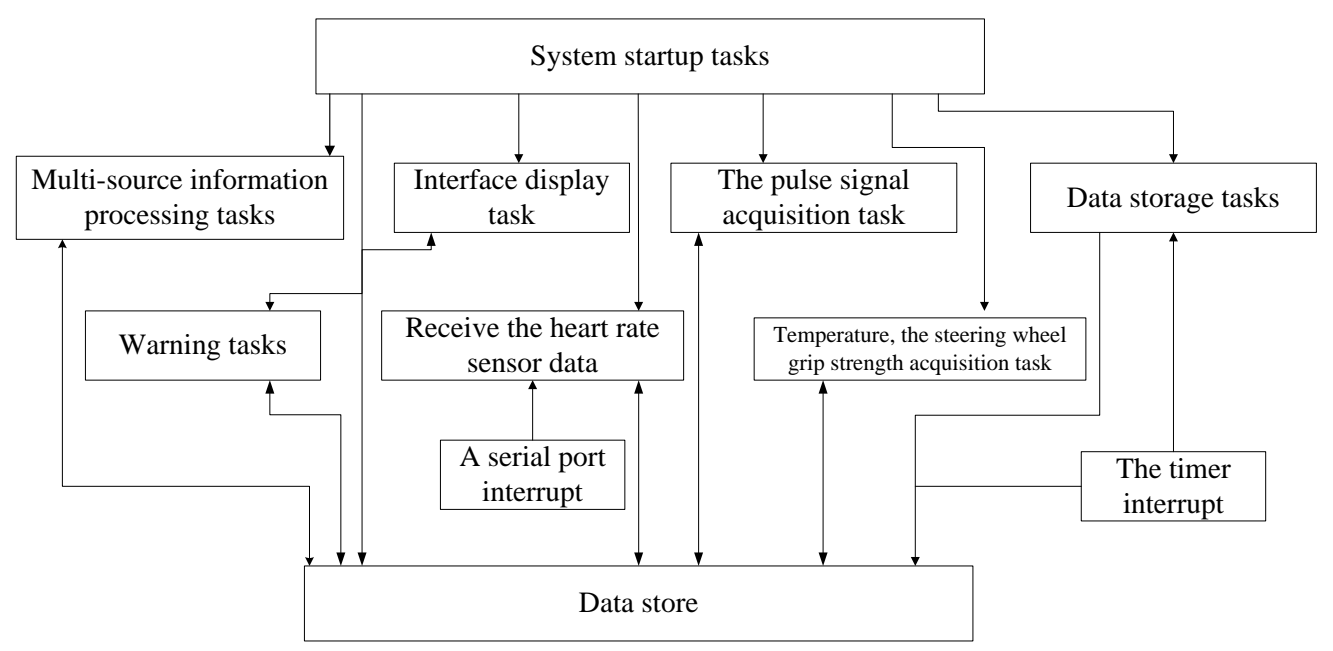

Fig.2 Diagram of system structure

\section{Realization of fatigue criterion}

When programming in the embedded software, reasonable distribution of the embedded system application task is a very important content, The reasonable design of the task will enable software have efficient code quality.

Otherwise, it will complicated the software code. The reasonable design will simplify the writing of the operating system, and the demand for system resources will be minimum.

\section{Software design}

UCOS - III operating system transplant.in order to facilitate transplantation, UCOS - III most of the code is written in $\mathrm{C}$, when it needs transplantation, it still need to be written in C language and assembly language code, when it related to processing hardware such as CPU registers operation code,it only can be written in assembly language. Transplanting UCOS - III mainly need to write or modify the three kind of CPU type kernel file: (os_cpu.h, os_cpu_c.c, os_cpu_a.asm), Os_cpu.h header file contains macros and functions related to the processor prototype statement; Os_cpu_a.asm file contains all the assembly language functions; Os_cpu_c.c contains the 
underlying operating functions, it is the core part of the system. UCGUI is a graphical support for embedded application software, it can work in multitasking system environment.

\section{D-S set of legal evidence}

D-S evidence theory first put forward by Dempster, Development by Shafer it is an imprecise theory, as well as an extension of the Bayes method, it does not require any prior information and conditional probability. In Bayes experiment, when the goal incompatible and there is no uncertainty measure, D-S evidence theory and Bayes method will produce the same results. In a multiple sensor information fusion system, each sensor provides a set of objective evidence, and establish the corresponding quality distribution function, in this way, each sensor can be seen as a body of evidence. Under the same criterion system, let the different rules of evidence use D-S to merge, so we forming a new body of evidence, and calculating the verisimilitude of evidence body, finally it determine the decision rules and the fatigue level.

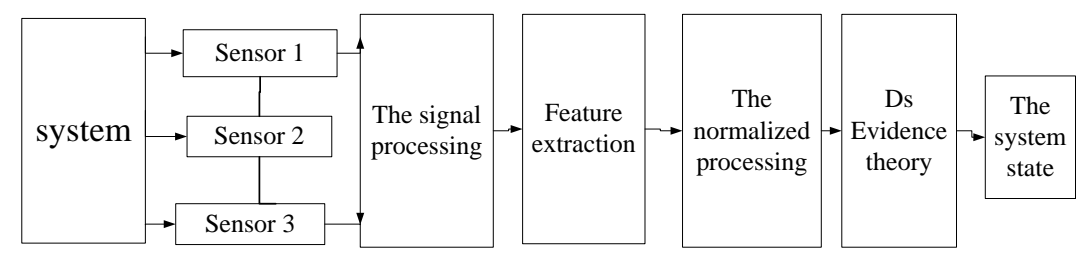

Fig.3 Figure of multi-source information fusion process

\section{The graphical interface design}

The interface of embedded fatigue early warning system is real-time display driver's physiological signal data, the LCD display interface of this system as shown in figure 4, The interface is mainly shows that the pulse ,body temperature, heart rate, the size of the steering wheel grip strength and driver's current state of fatigue. Detailed display design as follows: in the upper right corner is the display real-time of current date and time, In the screen above show that early warning system of the title "Driver Fatigue Monitor System" the central of screen is shows the "Pulse", "Temperature", "Heart rate" and "Grip" real-time data; Below display screen is fatigue State information, when the driver in the waking State, the green light on the board up and fatigue status appear two green signal; When the driver is in a state of slight fatigue, the yellow light on the onboard, fatigue status is displayed at the same time increase the two yellow signal to remind the driver is in slight fatigue; When the driver is in a severe fatigue, the red light flashing on the board and alarm speakers, and fatigue state shows two red signal to increase heart driver has been in a state of severe fatigue, measures must be taken to terminate the fatigue driving.

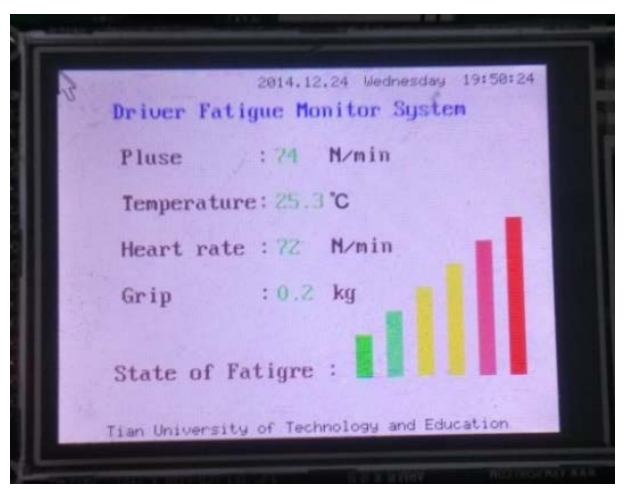

Fig.4 Warning system display interface 


\section{Conclusion}

By using the method of multi-source information fusion, this paper mainly completed the development of car driver fatigue early warning system based on embedded structure, it chose the ARM STM32F407 processor with DSP computation capability and combined with multitasking ability UCOS - III real-time operating system to realize the function of early warning system, Finally, according to the fatigue early warning system for the actual functional requirements, it designed main task of system, and introduced the working process of the task; finally early warning system for graphic display interface is designed.

\section{Acknowledgements}

This research is supported by the National Science Foundation for Young Scientists of China (601301040).

\section{Reference}

[1] Zhao Zhong. The driver fatigue detection method research and the realization of embedded [D]. Liaoning, Dalian Maritime University, 2010.

[2]Sun wei, Zhang wei gong, Zhang xiao-rui, Lv Chengxu, Chen gang. The research progress of fatigue test method [J]. Journal of automotive technology, 2009, 11:1-5.

[3] Li duhou, Liu qun, Yuan wei:, Liu haoxue Relationship between fatigue and traffic accident [J]. Journal of transportation engineering, 2010, 11:104-109.

[4] Yu tao, Du fudan CAN application in the automobile electronic [J]. Journal of electronic components in the world, 2006, (11) : 58-60.

[5] Xiao ShengBing Xiao hongju. UCOS-II on the ARM architecture (M3 processor transplantation. Electronics, 2010, (7) : 54-55.

[6] Han Chenghao Gao xiao-hong. CAN bus technology and its application [J]. Journal of manufacturing automation, 2010, 11:146-149.

[7] Dinges D F, Grace R. PERCLOS: A valid psychophysiological measure of alertness as assessed by psychomotor vigilance[R].Federal Highway Administration Office of Motor Carriers,1998.

[8] Fang Hengyao. Based on the research of GUI technology of automobile instrument development platform. Hubei: wuhan university of technology, 2007.

[9] Wang qian, Miao dehua, Deng san peng, Jiang yongxiang, Qi Yuming. Car driver fatigue monitoring method based on rough set study [J]. Vehicle and power technology, 2011, 2011:18- 21. 